\title{
A Novel Extreme Learning Machine for Hypoglycemia Detection
}

\author{
Phyo Phyo San, Sai Ho Ling Senior Member, IEEE, Ni Ni Soe, and Hung T. Nguyen Senior Member, IEEE
}

\begin{abstract}
Hypoglycemia is a common side-effect of insulin therapy for patients with type 1 diabetes mellitus (T1DM) and is the major limiting factor to maintain tight glycemic control. The deficiency in glucose counter-regulation may even lead to severe hypoglycaemia. It is always threatening to the well-being of patients with T1DM since more severe hypoglycemia leads to seizures or loss of consciousness and the possible development of permanent brain dysfunction under certain circumstances . Thus, an accurate early detection on hypoglycemia is an important research topic. With the use of new emerging technology, an extreme learning machine (ELM) based hypoglycemia detection system is developed to recognize the presence of hypoglycemic episodes. From a clinical study of 16 children with T1DM, natural occurrence of nocturnal hypoglycemic episodes are associated with increased heart rates $(p<0.06)$ and increased corrected QT intervals $(p<0.001)$. The overall data were organized into a training set with 8 patients (320 data points) and a testing set with 8 patients $(269$ data points). By using the ELM trained feed-forward neural network (ELM-FFNN), the testing sensitivity (true positive) and specificity (true negative) for detection of hypoglycemia is 78 and $60 \%$ respectability.
\end{abstract}

\section{INTRODUCTION}

Hypoglycemia, or low blood glucose levels, is one of the most common side effects among individuals with Type 1 diabetes. It is also well know as a barrier for achieving and maintaining tight glycemic control. Individuals who experience frequent episodes of hypoglycemia and those who have been on insulin therapy for a long period of time may develop impaired awareness of hypoglycemia. Impaired hypoglycaemic awareness occurs in approximately $25 \%$ of individuals with type 1 diabetes. Severe hypoglycemia may lead to physical and psychosocial morbidities such as brain damage, loss of consciousness, depression and low selfesteem [1]. It is also well known as death-in-bed syndrome [10].

To prevent or minimize hypoglycemic morbidities and mortalities, it is vital to return the blood glucose level to normal soon after a hypoglycemic event occurs. The patients' conditions are obviously need to know; when the event of hypoglycemia is initiated, and/or how the early warning is detected [3]. In other words, the need for hypoglycemia detection device is essential in order for giving alarm alert

The authors would like to thank Dr. Nejhdeh Ghevondian, and Assoc. Prof. Timothy Jones for their contribution. This works was supported by a grant from Juvenile Diabetes Research Foundation International.

Phyo Phyo San, Sai Ho Ling $^{1}$, and Hung T. Nguyen are with the Centre for Health Technologies, Faculty of Engineering and IT, University of Technology Sydney, Ultimo, NSW, Australia. $\quad{ }^{1}$ Steve.Ling@uts.edu.au; phone: +61 (2) 95142390

$\mathrm{Ni} \mathrm{Ni}$ Soe is with the School of Electrical and Electronic Engineering, Nanyang Technological University, Singapore. to Type 1 diabetes patients or carer when low blood glucose level is detected.

To analyze the blood glucose level (BGL) as well as the presence of hypoglycemia, many monitoring systems have been developed to be invasive or minimally invasive with the use of a needle-type probe [4]. However, this kind of measurement can only provide an isolated glucose level which does not reflect variations occurring throughout the day and night. For serve hypoglycemia, monitoring BGL continuously becomes almost impossible task, especially during the stage of nocturnal hypoglycemia.

Other minimally invasive devices [6] or non-invasive devices [7] have been tested and introduced with the aid of current technologies such as reverse iontophoresis, suction effusion fluid and near-infrared spectroscopy. But they suffer from limitations in terms of measurement inaccuracy, high susceptibility to artefact noise, considerable time delays for obtaining results and long-term reproducibility. The complications which are inherent with these techniques limit their use as practical non-invasive hypoglycemic monitors.

An alternative idea and solutions are still needed for noninvasive continuous monitoring of BGL as well as for prediction of hypoglycemia. To get a hold of this requirement, hypoglycemia detection systems have been investigated by the use of physiological parameters that are stimulated by falling blood glucose levels. For instance, Teledyne Sleep Sentry [8], multiparameter measurement system, Mini Med Medtronic CGMS and Abbott Freestyle Navigator CGMS [11].

Based on the strong relationship between physiological parameters and hypoglycemia, a non-invasive hypoglycemia monitor was tested and introduced by the use of the physiological parameters (heart rate (HR), corrected QT interval (QTc) and skin impedance) [12] [13]. By employing Bayesian neural network algorithm, the obtained sensitivity was found to be satisfactory, but the specificity need to be improved. More advanced neural network algorithms are still needed for obtaining better testing sensitivity and specificity. In addition, fuzzy estimator [14], fuzzy neural network estimator [15] were proposed for hypoglycemia detection systems. In these systems, the status of hypoglycemia was predicted through the changes in physiological parameters of the ECG signal. However, the membership functions which express the linguistic terms for fuzzy inference rules have to be defined. In practise, there is no formal approach for defining these rules and the optimization of these membership functions in terms of generalizing the data is also very important. Usually, tuning parameters of membership function is a time consuming task. 
In [16] [17], an evolved fuzzy reasoning model was developed to recognize the presence of hypoglycemic episodes. The physiological parameters such as heart rate and corrected QT interval of the electrocardiogram (ECG) signal were continuously measured for early detection of hypoglycemic episodes in Type 1 diabetes mellitus (T1DM) patients. In evolved FRM, the fuzzy rules and fuzzy membership functions were optimized by an evolutionary algorithm called hybrid particle swarm optimization with wavelet mutation (HPSOWM). The results showed that the proposed algorithm performed well in terms of the clinical sensitivity and specificity. However, in evolved FRM, more parameters were need to be optimized. Hence, it is desirable to have an automatic adaption procedure with less design parameters

Further experiment were conducted by the use of neural network based rule discovery system [18]. The development was based on 420 data sets which were collected from 16 T1DM patients by using the genetic algorithm. Though the obtained sensitivity and specificity are reasonable, the neural network used in the developed detection system might not be able to improve further because its slow learning speed and poor computational scalability.

To overcome some challenges faced by the mentioned state-of-the-art computational intelligence techniques, an emerging new technique named as extreme learning machine (ELM) [20] has been brought to the considerable attention. Generally, ELM is used to train for single-hidden layer feedforward neural network (FFNN). Different from common understanding of learning algorithm for FFNN, its hidden node parameters are randomly generated and no need to be tuned. Besides, it able to achieve the smallest training error as well as the smallest norm of output weights. Due to its significant features including faster learning speed and better generalization, in this paper, an ELM based hypoglycemia detection is developed.

The organization of this chapter is as follows: in Section II, a single layer feedforward neural network (FFNN) and an new emerging training algorithm, extreme learning machine (ELM) is introduced. To show the effectiveness of our proposed methods, the results of early detection of nocturnal hypoglycaemia episodes in T1DM are discussed in Section III and a conclusion is drawn in Section IV.

\section{METHODS}

To recognize the status of hypoglycemic episodes in T1DM, an ELM based hypoglycemia detection system in Fig. 2 is developed by the use of physiological parameters of ECG signal Fig. 1.

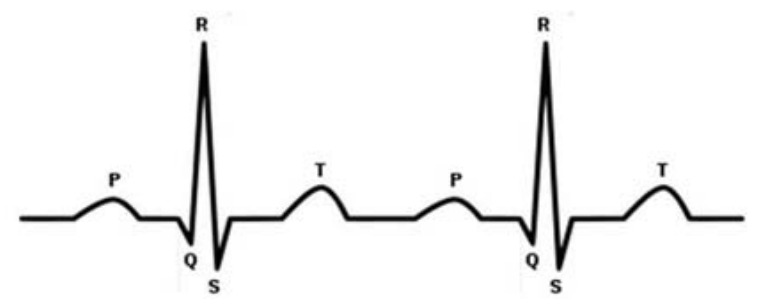

Fig. 1. ECG Signal
For patient with Type 1 and Type 2 diabetes, the possibility of hypoglycemia is mainly effected by prolongation of QT intervals (starting from the point of $\mathrm{Q}$ wave to at the end of $\mathrm{T}$ wave) and its correlation to heart rate is carried out by Bazett's formula $Q T_{c}=Q T / R R$ [19] . Not only $Q T_{c}$ interval prolongation has a significant impact on hypoglycemia, but an increase in heart rate (HR) also may influence the status of hypoglycemia. With these change of physiological parame-

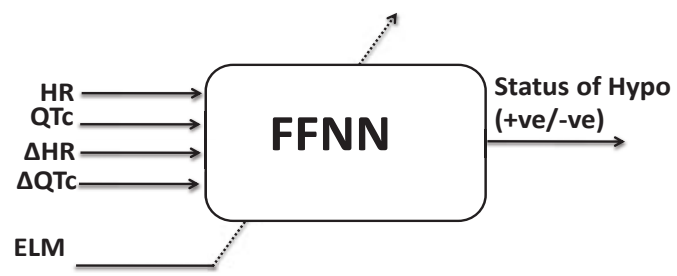

Fig. 2. ELM based hypoglycemia detection system

ters, in this paper, an ELM based hypoglycemia detection system is developed for early detection of hypoglycemic episodes. The proposed ELM based model with four inputs such as heart rate (HR), corrected QT interval $\left(Q T_{c}\right)$, change of heart rate and corrected QT interval, $(\Delta H R)$ and $\left(\Delta Q T_{c}\right)$ and one output system (hypoglycemia status $(h)$ ) is developed as shown in Fig. 2.

\section{A. Extreme Learning Machine (ELM)}

In this section, extreme learning machine (ELM) for the training of single hidden layer feedforward neural network (FFNN) in Fig. 3 is presented. Compared with the conventional neural network learning algorithms, it can achieve faster training performance and overcome the problem of over-fitting. The algorithm is based on the empirical risk minimization theory. Its learning process needs only for a single iteration and is able to avoid multiple iterations and local minimization. Because of better generalization ability, robustness, and controllability and faster learning process, it has been used in many real world applications [21]. For $N$ arbitrary distinct samples $\left(x_{i}, t_{i}\right) \in R^{d} \times R^{m}$, the mathematically model of FFNN with $L$ hidden nodes can be written as:

$$
y_{j}=\sum_{i=1}^{L} \beta_{i} g_{i}\left(x_{j}\right)=\sum_{i=1}^{L} \beta_{i} G\left(a_{i}, b_{i}, x_{j}\right), \quad j=1, \ldots, N
$$

The FFNN can approximate these $N$ samples with zero error means that $\sum_{i=1}^{L}\left\|y_{j}-t_{j}\right\|=0$, i.e., there exist $\left(a_{i}, b_{i}\right)$ and $\beta_{i}$ such that $\sum_{i=1}^{L} \beta_{i} G\left(a_{i}, b_{i}, x_{j}\right)=t_{j}$, whereas $j=1, \ldots, N$. It can be expressed as:

$$
H \beta=T
$$

where

$$
H=\left[\begin{array}{c}
h\left(x_{1}\right) \\
\vdots \\
h\left(x_{N}\right)
\end{array}\right]=\left[\begin{array}{ccc}
G\left(a_{1}, b_{1}, x_{1}\right) & \ldots & G\left(a_{1}, b_{1}, x_{1}\right) \\
\vdots & \cdots & \vdots \\
G\left(a_{1}, b_{1}, x_{1}\right) & \cdots & G\left(a_{1}, b_{1}, x_{1}\right)
\end{array}\right]
$$




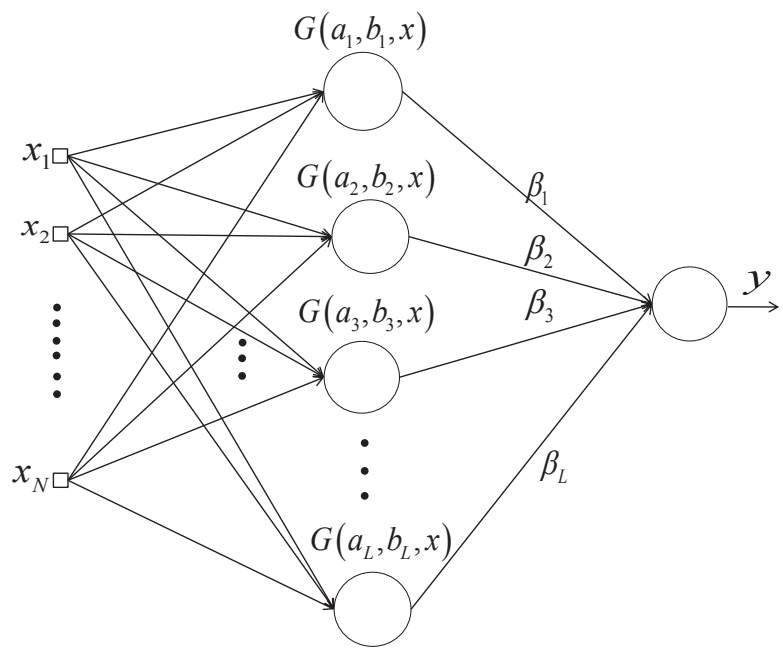

Fig. 3. Feedforward neural network with single hidden layer

$$
\beta=\left[\begin{array}{c}
\beta_{1}^{T} \\
\vdots \\
\beta_{L}^{T}
\end{array}\right]_{L \times m}, \quad T=\left[\begin{array}{c}
t_{1}^{T} \\
\vdots \\
t_{N}^{T}
\end{array}\right]_{N \times m}
$$

$H$ is called the hidden layer output matrix of the FFNN, the $i$ th column of $H$ is the $i$ th hidden node output with respect to inputs $x_{1}, x_{2}, \ldots, x_{N}, h(x)=G\left(a_{1}, b_{1}, x\right) \ldots G\left(a_{L}, b_{L}, x\right)$ is called as the hidden layer feature mapping. From the interpolation capability point of view, if the activation function $G$ is infinitely differentiable in any interval the hidden layer parameters can be randomly generated [20]. It means that the hidden layer output matrix $H$ in (3) is initialized with random weight $\left(a_{i}\right)$ and bias $\left(b_{i}\right)$ and calculate the output weight matrix $\beta$ from the linear equation in (2) by:

$$
\beta=H^{\dagger} T
$$

where $H^{\dagger}$ is the Moore-Penrose generalized inverse of the matrix $H$. To obtain $H^{\dagger}$, the orthogonal projection method can be efficiently used, whereas $H^{\dagger}=\left(H^{T} H\right)^{-1} H^{T}$ (or) $H^{\dagger}=H^{T}\left(H H^{T}\right)^{-1}$ if $H^{T} H$ and $H H^{T}$ are nonsingular. Substituting the output weight matrix in (1) to (5) and calculate the corresponding output of FFNN. The proposed hypoglycemia detection system, FFNN trained by ELM algorithm is given in detail as follows:

1) The hidden node parameters, $\left(a_{i}, b_{i}\right), i=1, \ldots, L$ are randomly generated.

2) The hidden layer output matrix $H$ in (3) is calculated.

3) Obtain the output weight vector $\beta$ in (5) by $\beta=$ $\left(H^{T} H\right)^{-1} H^{T} T$ (or) $\left(H H^{T}\right)^{-1} H^{T} T$.

4) The output of FFNN , $y$ is calculated by (1) and it is defined as positive when the status of hypoglycemia $h$ is positive which expressed as:

$$
h=\left\{\begin{array}{cc}
+1, & y \geq 0 \\
-1, & y<0 .
\end{array}\right.
$$

According to conventional neural network theories, hidden layer parameters, ie., weight and biases $\left(a_{i}\right.$ and $\left.b_{i}\right)$ and the output weights $\beta_{i}$ need to be tuned properly and searching for optimal parameters. Much efforts have been tried for obtaining better way of finding and adjusting hidden layer parameters $\left(a_{i}, b_{i}\right)$. In [22], incremental learning method which mainly focus and adjust on the newly added hidden nodes rather than adjusting all the hidden nodes parameters. In contrast, all the parameters of FFNN such as weight $\left(a_{i}\right)$ and biases $\left(b_{i}\right)$ and the output weight parameters $\left(\beta_{i}\right)$ need not be tuned and can be independent of training samples.

Similar to the conventional neural network, the numbers of hidden nodes are chosen by trial and error method until the optimal solution is obtained. However, there is a trade-off between too less number and too many numbers of hidden nodes because less number of node is insufficient to give the optimal solution and more numbers may unnecessarily increase the number of parameters to be tuned by ELM.

In essence, the ELM need to be iteratively tuned and both the training error and the norm of weight can be minimized as long as the hidden layer feature mapping need to satisfy approximation condition [23]. In ELM, the hidden nodes can be randomly generated and the output weights vector $\beta_{i}$ is the only unknown parameters which can be resolved by ordinary least-square directly. Unlike the traditional computational intelligence techniques, ELM also provide better generalization performance at a much faster convergence rate without over-fitting problem.

\section{RESULT AND DISCUSSION}

In this group of study, all 16 patients (children with ages $14.6 \pm 1.5$ years) were Type 1 diabetes patients and they underwent for overnight electrocardiogram (ECG) and glucose monitoring at the Princess Margaret Hospital for Children in Perth, Australia. For each patient, all the data were collected between 360 minutes to 480 minutes and approximately 35-40 data points were collected for a sampling period of between 5 and 10 minutes.

The relationships between overnight measurements of QT interval corrected for heart rate (QTc) and falling BGL were discovered by the use of statistical correlation analysis. The overall data set of 16 children with Type 1 diabetes [12] were considered in this study due to their significant increment in HR and QTc in response to episodes of hypoglycemia (1.033 \pm 0.242 vs. $1.082 \pm 0.242 \pm p<0.06)$ and $(1.031 \pm 0.086$ vs. $1.060 \pm 0.084 p<0.001)$. To perform the classification test, the overall data set consisted of a training set with 8 patients (320 data points) and a testing set with 8 patients (269 data points), randomly selected.

For comparison and analysis purposes, five methods are applied to the hypoglycemia detection problem as following:

1. Proposed feed-forward neural network trained with ELM with 15 hidden nodes (FFNN-ELM);

2. Feed-forward neural network trained with particle swarm optimization (FFNN-PSO)[17];

3. Second order multiple regress fuzzy inference system 
(MR-FIS) [16];

4. Fuzzy inference system with 5 membership functions for each input (FIS) [17];

5. Linear multiple regression (LMR)[16].

All of the methods are applied into the hypoglycemia detection system where the four inputs of the system are heart rate (HR), QT interval (QTc), $\triangle H R$ and $\Delta Q T c$ and one output to represent the onset of hypoglycemia. The testing results in term of sensitivity and specificity are tabulated in Table I. Moreover, the training time for all methods are also shown in the same table to illustrate how fast of the proposed method compared with other methods. In Table I, we can seen that the testing sensitivity and specificity of proposed ELM based FFNN achieve 78 and $60 \%$ which is better than $75.00 \%$ testing sensitivity and $50.23 \%$ specificity given by multiple regression with fuzzy inference system (MRFIS) [16]. Also, compared with FFNN-PSO which is trained PSO, the testing performance of the FFNN trained by ELM is outperformed. Besides, the proposed FFNN-ELM gives a faster training time which is about 3 mins, while the other methods (FFNN-PSO, MR-FIS, and FIS) take around 30 to 275 mins. LMR gives a very fast training time, however, the testing performance is unacceptable. In Summary, the FFNNELM gives satisfactory results with faster training time.

TABLE I

TESTING Results AND TRAINING TIME

\begin{tabular}{|l|c|c|c|}
\hline Method & Sensitivity & Specificity & Training time \\
\hline FFNN-ELM & $78.00 \%$ & $60.00 \%$ & $3 \mathrm{mins}$ \\
\hline FFNN-PSO & $63.64 \%$ & $54.67 \%$ & $30 \mathrm{mins}$ \\
\hline MR-FIS & $75.00 \%$ & $50.23 \%$ & $40 \mathrm{mins}$ \\
\hline FIS & $75.00 \%$ & $51.64 \%$ & $275 \mathrm{mins}$ \\
\hline LMR & $51.78 \%$ & $51.64 \%$ & $1 \mathrm{~min}$ \\
\hline
\end{tabular}

\section{CONCLUSIONS}

For detection of the hypoglycemic episodes for diabetes patients an ELM based diagnosis system has been developed. The above results indicate that hypoglycemic episodes in T1DM children can be detected non-invasively and effectively by the use of real-time physiological parameters of ECG signal. The performance of proposed detection system is evaluated with other comparison methods and the improvement in sensitivity can be satisfactorily found with faster training time.

\section{REFERENCES}

[1] L. G. Frederick and J. Zrebiec, "Detection of hypoglycemia by children with type 1 diabetes 6 to 11 years of age and their parents: a field study", Pediatric, vol. 121, no. 3, pp. e489-e495, 2008.

[2] D. C. Klonoff, "The need for hypoglycemia detection and prevention in type 1 diabetes", Diabetes Technology and Therapeutics, vol. 3, no. 4, pp. 567-570, 2001.

[3] A. Osareh and B. Shadgar, "Intrusion detection in computer networks based on machine learning algorithms", International Journal of Computer Science and Network Security, vol. 8, no. 11, pp. 15-23, 2008.
[4] K. Kajiwara and T. Uemura and H. Kishikawa, "Noninvasive measurement of blood glucose concentrations by analysing Fourier transform infra-red absorbance spectra through oral mucosa", Medical and Biological Engineering and Computing, vol. 31, no. 8, pp. S17-S22, 1993.

[5] J. Kimura and N. Ito and T. Kuriyama, "A novel blood glucose monitoring method", Chemical Sensors, vol. 87, no. 9, pp. 327-333, 1987.

[6] J. Kimura and N. Ito and T. Kuriyama, "A novel blood glucose monitoring method", Chemical Sensors, vol. 87, no. 9, pp. 327-333, 1987.

[7] H. M. Heise and R. Marbach and T. Koschinsky, "Noninvasive blood glucose sensors based on near-infrared spectroscopy", Artificial Organs, vol. 18, no. 6, pp. 439-447, 1994.

[8] J. Castano and Y. Z. Wang and D. Chuang, "Blood glucose dependence of visual flicker threshold", Diabetes Technology and Therapeutics, vol. 2, no. 1, pp. 31-43, 2000.

[9] F. F. R. Maia and L. R. Arajo, "Nocturnal hypoglycemia in patients with insulin-treated diabetes", Diabetes Research and Clinical Practice, vol. 75, no. 1, pp. 30-34, 2007.

[10] D. C. Klonoff, "The need for hypoglycemia detection and prevention in type 1 diabetes", Diabetes Technology and Therapeutics, vol. 3, no. 4, pp. 567-570, 2001.

[11] R. L. Weinstein, "Accuracy of the 5-day FreeStyle Navigator Continuous Glucose Monitoring System: comparison with frequent laboratory reference measurements", Diabetes Care, vol. 30, no. 5, pp. 11251130, 2007.

[12] H. T. Nguyen and N. Ghevondian and T. W. Jones, "Neural-network detection of hypoglycemic episodes in children with type 1 diabetes using physiological parameters", 28th Annual International Conference of the IEEE Engineering in Medicine and Biology Society, pp. 6053-6056, 2006.

[13] H. T. Nguyen and N. Ghevondian and S. T. Nguyen and T. W. Jones, "Detection of hypoglycemic episodes in children with type 1 diabetes using an optimal Bayesian neural network algorithm", International Conference of IEEE Engineering in Medicine and Biology Society, pp. 3140-3143, 2007.

[14] N. Ghevondian and H. T. Nguyen, "Using fuzzy logic reasoning for monitoring hypoglycemia in diabetic patients", 19th Annual International Conference of the IEEE Engineering in Medicine and Biology Society, pp. 1108-1111, 1997.

[15] N. Ghevondian and H. T. Nguyen, "A novel fuzzy neural network estimator for predicting hypoglycaemia in insulin-induced subjects", 23rd Annual International Conference of the IEEE Engineering in Medicine and Biology Society, pp. 1657-1660, 2001.

[16] S. H. Ling and H. T. Nguyen, "Genetic-algorithm-based multiple regression with guzzy inference system for detection of nocturnal hypoglycemic episodes", IEEE Transactions on Information Technology in Biomedicine, vol. 15, no. 2, pp. 308-315, 2011.

[17] S. H. Ling and H. T. Nguyen, "Natural occurrence of nocturnal hypoglycemia detection using hybrid particle swarm optimized fuzzy reasoning model", Artificial Intelligence In Medicine, vol. 55, no. 3, pp. 177-184, 2012.

[18] K. Y. Chan, S. H. Ling, T. S. Dillon and H. T. Nguyen, "Diagnosis of hypoglycemic episodes using a neural network based rule discovery system", Expert Systems with Applications, vol. 38, no. 8, pp. 97999808, 2011.

[19] V. Srinivasan and C. Eswaran and N. Sriraam, "Approximate entropybased epileptic EEG detection using artificial neural networks", IEEE Transactions on Information Technology in Biomedicine, vol. 11, no. 3, pp. 288-295, 2007.

[20] G. B. Huang, D. H.Wang, and Y. Lan, "Extreme learning machines: a survey", International journal of machine learning and cybernetics, vol. 2, pp. 107-122, 2011

[21] G. B. Huang, Q. Y. Zhu, and C. K. Siew, "Extreme learning machine: theory and applications", Neurocomputing, vol. 70, pp. 489-501, 2006.

[22] A. R. Barron, "Universal approximation bounds for superpositions of a sigmoidal function", IEEE Transactions on Information Theory, vol. 39, no. 3, pp. 930-945, 1993.

[23] G. B. Huang, H. Zhou, X. Ding, and R. Zhang, "Extreme learning machine for regression and multiclass classification", IEEE Transactions Systems, Man, and Cybernetics, Part B, vol. 42, pp. 513-529, 2012. 